\title{
How Family Physicians Distinguish Acute Sinusitis From Upper Respiratory Tract Infection: A Retrospective Analysis
}

\author{
David R. Little, MD, MS, Barbara L. Mann, PbD, and Cbristopher J. Godbout, MD
}

Background: The purpose of our study was to examine how physicians diagnose sinusitis in practice. We addressed three specific questions: (1) what clinical factors do physicians look for in evaluating and caring for patients with suspected sinusitis, (2) to what extent do physicians use transillumination and radiograph evaluations in diagnosing sinusitis, and (3) how does the diagnosis of sinusitis influence the decision to prescribe antibiotic therapy?

Methods: We conducted a retrospective review using charts from 25 local family physicians who volunteered to participate in the study. After selecting a random sample of charts of adult patients treated for sinusitis and for upper respiratory tract infection (URI) by each physician, we reviewed the charts to determine the nature of the information collected to differentiate between sinusitis and URI.

Results: Rhinorrhea, sinus tenderness, visualization of purulent secretions, and a history of sinusitis were significant predictors of the diagnosis of sinusitis. Antibiotics were prescribed for $98.4 \%$ of patients with sinusitis and $13.1 \%$ of patients with URI.

Conclusions: This sample of physicians based the diagnosis of sinusitis on three prominent clinical findings, which were also significant factors in diagnosing sinusitis in previous studies. The history of sinusitis might influence patient and physician expectations for the diagnosis. (J Am Board Fam Pract 2000;13:101-6.)

The diagnosis of acute bacterial sinusitis is frequently made on clinical grounds. A body of literature has examined the accuracy of the clinical diagnosis of sinusitis ${ }^{1-3}$ and the specific factors that influence the physician in making the diagnosis. ${ }^{4}$ Use of ancillary techniques, such as transillumination, ${ }^{5}$ radiograph examinations, ${ }^{6}$ sonography, ${ }^{7}$ computed tomographic scanning, ${ }^{8}$ and laboratory studies $^{9}$ have also been explored. The recent Agency for Health Care Policy and Research (AHCPR) evidence report on diagnosis and treatment of acute bacterial rhinosinusitis concluded, "limited evidence suggests that clinical criteria ... may have a diagnostic accuracy similar to that of sinus radiography." 10 The consensus has arisen from this literature that the clinical diagnosis of sinusitis is a valid approach.,11

The specific clinical factors physicians emphasize when diagnosing sinusitis have only recently

Submitted, revised, 5 October 1999.

From the Department of Family Medicine (DRL, BLM, CJG), Wright State University School of Medicine, Dayton, Ohio. Address reprint requests to David R. Little, MD, MS, Department of Family Medicine, WSU/SOM, One Franciscan Way, Dayton, OH 45408. begun to be examined. In a previous study ${ }^{4}$ the authors developed a series of simulated case histories based on clinical factors described in the literature on sinusitis. The cases were presented to a sample of family physicians to determine which factors most strongly influenced their decision to diagnose sinusitis and prescribe antibiotics. The study also examined the extent to which physicians reported the use of transillumination and radiograph examinations in the diagnosis of sinusitis.

The previous study found that the diagnosis of sinusitis is strongly influenced by the number of clinical factors present. Individual factors with a significant influence on the diagnosis included a history of maxillary or. facial pain, a history of colored nasal discharge, and lack of response to decongestants. Physical findings of sinus tenderness, purulent drainage visualized on nasal examination, and fever also significantly influenced the diagnosis. Each of these factors had a similar influence on the physicians' decision to prescribe antibiotics. Smoking history as a factor in the simulated cases had a minimal impact on the diagnosis and treatment of sinusitis. Sixty percent of the physicians in the study stated that they perform transil- 
lumination. The median estimated percentage of cases for which radiographs would be obtained was $6.25 \%$.

A recent study by Hueston and colleagues ${ }^{12}$ examined practice patterns of resident physicians in differentiating between sinusitis and upper respiratory tract infection (URD). Their report concluded that residents relied on unreliable clinical findings (sinus tenderness and sinus pressure) to diagnose sinusitis, with the possibility of overdiagnosis and overtreatment.

Although the use of simulated cases in the previous study provided insight into the medical decision-making process, it might not accurately reflect what physicians actually do in practice. The purpose of our study was to examine how experienced physicians diagnose sinusitis in practice. The following specific questions were addressed: (1) Which clinical factors do physicians look for in evaluating and treating patients with suspected sinusitis? (2) To what extent do physicians use transillumination and radiograph evaluations in diagnosing sinusitis? (3) How does the diagnosis of sinusitis influence the decision to prescribe antibiotic therapy?

\section{Methods}

We conducted a retrospective review using charts from 25 local family physicians who volunteered to participate in the study. Eight practice sites were represented. Five sites were faculty practices, incorporating 20 faculty physicians from the Wright State University Department of Family Medicine and its affiliated residencies. Three private practices representing 5 family physicians were also included. We selected from each physician a random sample of 10 charts of adult patients treated for an acute episode of sinusitis during calendar year 1995. We also chose from each physician a control sample of 10 charts of patients treated for URI. We selected this group on the assumption that the physicians applied the diagnosis of URI to patients with a presumed viral cause for their symptoms.

We excluded charts from the sinusitis group if the patient had an established diagnosis of chronic sinusitis, because it might change the approach to diagnosis and management. If a chart showed that several office visits took place to resolve a single episode of sinusitis, then we analyzed only the ini- tial office visit that resulted in the diagnosis of sinusitis. We also excluded charts from the URI group if additional diagnoses implicated possible bacterial infection (ie, URI with bronchitis, otitis media, and so on).

We reviewed the charts to determine the nature of the information recorded to establish a diagnosis. The following factors were specifically examined:

1. Historical information, including rhinorrhea, headache, facial pain, report of fever, response to decongestants, recent URI, history of sinusitis, and smoking history. If rhinorrhea was reported, any report of discolored nasal discharge was also sought

2. Physical examination findings, including temperature, general appearance, sinus tenderness, discolored drainage observed on nasal examination, pharynx examination findings, and lymphadenopathy

3. Additional studies performed, including transillumination and radiograph examinations, and the results of these studies

4. The decision whether to prescribe an antibiotic

We considered each clinical factor to be present if a positive finding was recorded in the chart. If a factor was recorded as negative or not recorded in the progress note, we considered that factor to be absent.

We reviewed smoking history by examining the progress note and the problem list at the front of the chart, if available. We categorized charts into three groups: smokers, documented nonsmokers, and smoking history not recorded.

\section{Data Analysis}

We tabulated patient demographics for sinusitis and control groups and compared using the Fisher exact test and $t$ tests.

As a follow-up to the simulated cases study by Little et al, ${ }^{4}$ we used logistic regression to compare the two diagnostic groups for differences on a total of 15 factors after adjusting for physician effect. Few charts had notations of colored nasal discharge reported by the patients, so for this variable we substituted a history of rhinorrhea in the analysis.

We compared the rates of prescribing antibiotics for the sinusitis and control groups using chisquare statistics. We did not consider it to be nec- 
essary to have logistic regression models for antibiotic prescribing, because the prescribing behavior closely paralleled that for the diagnosis of sinusitis.

Smoking behavior presented a special problem in the logistic regressions because of the relatively high proportion of charts that had no record of smoking status. Technically these charts should produce missing values for the smoking variable. Because a missing value for one variable leads to exclusion of the entire case from a logistic regression, a new variable was created for these analyses that lumped together the values of nonsmoking and missing. Thus we misclassified as nonsmokers an unknown percentage of patients who were smokers. The effect of this misclassification on the analysis was to make it more difficult to detect an effect of smoking behavior on the response variables. As a follow-up analysis, we used the Fisher exact test to compare the proportions of smokers and nonsmokers in the two diagnosis groups using only the data for which smoking behavior appeared in the patient record.

A logistic regression analysis also included the total number of factors as a predictor for diagnosis. To avoid problems in this analysis, it was necessary to drop the least significant variable (lack of response to decongestants) from the list of variables used.

We divided duration of illness into quartiles for the pooled data, then used a chi-square test to test for differences in this variable between the sinusitis and URI groups. We cross-classified data on radiographs, transillumination, and antibiotic use by diagnostic group and analyzed them using the Fisher exact test.

\section{Results}

A total of 392 charts met the inclusion criteria and were reviewed for the study. This sample included 193 patients with sinusitis and 199 with URI. In some cases, the target number of 10 charts per physician in each group was not available. One faculty practice site was relatively new and had not accumulated a large number of office visits at the time of the study. Three other physicians had office visits that were skewed toward one diagnosis or the other, so a complete sample of 10 charts from each group was not available.

We compared sinusitis and control groups by patient age, race, and sex (where available), with the
Table 1. Patient Demographics.

\begin{tabular}{lccl}
\hline Variable & $\begin{array}{c}\text { Sinusitis } \\
(\mathrm{n}=193)\end{array}$ & $\begin{array}{c}\text { Control } \\
(\mathrm{n}=197)\end{array}$ & $P$ Value Test \\
\hline Age, years & & & \\
$\quad$ Mean & 40.6 & 38.9 & NS \\
$\quad$ Standard deviation & 12.8 & 16.2 & $t$ test \\
Race & 12 & 35 & NS \\
$\quad$ White & 3 & 5 & Chi-square \\
Black & 3 & 3 & \\
Other & $\cdot$ & & \\
Sex & 54 & 60 & NS \\
Male & 139 & 137 & Chi-square \\
Female & & & \\
\hline
\end{tabular}

NS-Not significant.

results displayed in Table 1 . There were very few missing data for age and sex, but the patient's race was often not recorded in the chart. There were no significant differences between these two groups on any of the demographic variables.

The logistic regression results for diagnosis of sinusitis appear in Table 2. Consistent with the findings of Little et al, ${ }^{4}$ purulent drainage and sinus tenderness as observed by the physician, along with rhinorrhea reported by the patient, were significant predictors of the diagnosis of sinusitis. A patientreported history of sinusitis, abnormal transillumination results, and facial pain were also significant predictors.

When we included the number of positive signs and symptoms in the logistic regression models, the actual number did not have a significant effect on the diagnosis of sinusitis. When we restricted the analysis to include only the specific signs and symptoms described in the simulated case histories, the results still showed no significant effect. The relative significance of the individual factors did not change appreciably in the presence of this summary variable. The duration of illness also did not achieve statistical significance in differentiating between sinusitis and URI.

Smoking histories were recorded in 158 of the 392 cases (40.3\%). Among patients with a documented smoking history, $60(38.0 \%)$ were reported as current smokers and $98(62.0 \%)$ as nonsmokers. The sinusitis group had a higher proportion of smokers (Table 3), but this result did not achieve statistical significance.

Transillumination was performed in only 24 of the 392 cases $(6.1 \%)$. Findings were reported as 
Table 2. Logistic Regression Analysis of Contributions of Individual Factors to the Diagnosis of Sinusitis.

\begin{tabular}{|c|c|c|c|c|c|}
\hline \multirow[b]{2}{*}{ Factor } & \multirow[b]{2}{*}{$P$ Value } & \multirow[b]{2}{*}{ Odds Ratio } & \multirow[b]{2}{*}{$95 \% \mathrm{CI}$} & \multicolumn{2}{|c|}{ Percent of Cases } \\
\hline & & & & URI & Sinusitis \\
\hline Discolored drainage visualized on examination ${ }^{*}$ & $.0001^{\dagger}$ & 28.286 & $(5.678,140.912)$ & 2.0 & 18.1 \\
\hline Sinus tenderness on examination ${ }^{*}$ & $.0001^{\dagger}$ & 10.977 & $(3.471,34.715)$ & 5.0 & 34.7 \\
\hline Rhinorrhea reported by patient ${ }^{*}$ & $.001^{\dagger}$ & 3.761 & $(1.710,8.273)$ & 33.2 & 46.1 \\
\hline History of sinusitis & $.006^{\dagger}$ & 3.118 & $(1.397,6.961)$ & 12.6 & 29.0 \\
\hline Abnormal transillumination & $.010^{\dagger}$ & 31.735 & $(2.278,442.095)$ & 0.5 & 6.2 \\
\hline Maxillary toothache or facial pain ${ }^{*}$ & $.020^{\ddagger}$ & 4.091 & $(1.235,13.550)$ & 4.0 & 21.2 \\
\hline Headache reported by patient & .0582 & 2.043 & $(0.976,4.280)$ & 15.6 & 31.6 \\
\hline Abnormal nasal examination & .0811 & 1.905 & $(0.924,3.930)$ & 30.7 & 48.7 \\
\hline History of smoking recorded in chart & .1179 & 1.882 & $(0.852,4.159)$ & 11.6 & 19.2 \\
\hline Recent URI & .2663 & 1.660 & $(0.679,4.059)$ & 10.0 & 9.3 \\
\hline Abnormal pharynx examination & .3069 & 1.379 & $(0.744,2.556)$ & 36.2 & 44.6 \\
\hline Fever $\left(>100^{\circ} \mathrm{F}\right)$ on examination ${ }^{*}$ & .6467 & 0.602 & $(0.069,5.260)$ & 1.0 & 3.0 \\
\hline Abnormal general appearance & .7060 & 0.574 & $(0.032,10.248)$ & 1.5 & 1.0 \\
\hline Abnormal lymph nodes on examination & .7130 & 1.237 & $(0.399,3.835)$ & 7.0 & 11.4 \\
\hline Lack of response to decongestants" & .7833 & 0.906 & $(0.447,1.836)$ & 22.1 & 24.4 \\
\hline
\end{tabular}

$\mathrm{CI}=$ confidence interval, URI = upper respiratory tract infection.

"Factors examined in previous study. ${ }^{4}$

'Significant at the $P .01$ level.

*Significant at the $P .05$ level.

abnormal in 13 of these 24 cases (54.2\%); of these abnormal findings, $12(92.3 \%)$ received a diagnosis of sinusitis. There were no radiograph examinations for patients in either group.

Analysis of antibiotic use showed that antibiotics were prescribed in 190 of the 193 cases of sinusitis (98.4\%), but only 26 of the 199 cases of URI $(13.1 \%)(P<.001)$.

\section{Discussion}

Four clinical factors found to be significant in this review are substantially in agreement with those found in previous studies. Physicians in this sample regarded facial pain, discolored rhinorrhea, sinus tenderness, and visualization of purulent discharge

Table 3. Influence of Smoking Status on Diagnosis.

\begin{tabular}{lcc}
\hline Status & $\begin{array}{c}\text { Sinusitis Group } \\
\left(\mathrm{n}=82^{*}\right. \\
\text { No. }(\%)^{\dagger}\end{array}$ & $\begin{array}{c}\text { URI Group } \\
(\mathrm{n}=76)^{*} \\
\text { No. }(\%)^{+}\end{array}$ \\
\hline Smoker & $37(45.1)$ & $23(30.3)$ \\
Nonsmoker & $45(54.9)$ & $53(69.7)$ \\
\hline
\end{tabular}

URI-upper respiratory tract infection.

*Numbers represent patients for whom smoking status was recorded.

${ }^{\dagger}$ Percentages are column percents.

$P=.071$. as factors strongly consistent with sinusitis. These four factors were also reported in the previous responses of a group of family physicians to a series of simulated cases of suspected sinusitis. ${ }^{4}$ With the exception of sinus tenderness, each of these factors was also found to be a predictor of sinusitis in the logistic regression analysis previously performed by Williams et al. ${ }^{2}$ Smoking history appeared to have a small effect that did not achieve statistical significance. This result is also quite similar to the effect of smoking in the simulated cases.

A new factor that arose from this study was the history of sinusitis. This factor had a significant effect on the diagnosis, although it was not considered in previous studies. The resident physicians studied by Hueston et al $^{12}$ also showed a significant tendency to diagnose sinusitis in a patient who had a history of sinus infections. Perhaps a subset of patients has anatomic, immunologic, or allergic problems that predispose them to recurrent sinusitis. Alternatively, a history of sinusitis might influence the expectations of the patient or the physician in such a way that the diagnosis of sinusitis and the antibiotic prescription become more likely. Previous studies of the treatment of respiratory tract infections have shown that rates of antibiotic use are significantly higher when physicians believe 
patients expect treatment, although receiving a prescription for antibiotics is not directly associated with increased patient satisfaction. ${ }^{13-15}$

There were several points where the factors in our study were not consistent with previous reports. Most notably, the number of factors recorded did not significantly affect the diagnosis of sinusitis in this study. Perhaps these physicians have a more targeted approach to the diagnosis, basing their decisions on the specific signs, symptoms, and historical features that they choose to record.

The study by Williams et $\mathrm{al}^{2}$ found the failure to respond to decongestants to be a predictor of sinusitis. This factor was not significant in this study and was less significant than other factors in the responses to the simulated cases. ${ }^{4}$

In the responses to the simulated cases, the finding of a fever on physical examination was significantly associated with the diagnosis of sinusitis. In the present study, only 8 of the 392 patients were found to be febrile, and only 2 of these 8 (25\%) had sinusitis diagnosed. Similarly, physicians responding on a Likert scale indicated that the duration of illness was important in diagnosing sinusitis, ${ }^{4}$ but this factor was not significant in the present study.

Transillumination was used sparingly in this sample. Only 10 of the 25 physicians (40\%) in this study recorded transillumination findings on any patient. This percentage is in contrast with the $60 \%$ of physicians who reported performing this procedure even intermittently in the previous study. No radiograph examinations or other imaging studies were performed in either group on this study, suggesting that these studies might be reserved for more recurrent or resistant cases of sinusitis.

Antibiotic use in patients diagnosed with sinusitis approached $100 \%$ in this sample of patients seen in 1995. With the growth of evidence-based medicine and the recent movement away from universal use of antibiotics for sinusitis, ${ }^{12,13}$ it will be interesting to monitor this figure in the years to come. That $13 \%$ of patients with a diagnosis of URI were treated with antibiotics raises additional questions for future study. Are there certain characteristics of these patients, across a larger population, that prompt the physician to recommend antibiotic therapy?

A final question raised by this study concerns the observation that 3 of the 25 physicians had large disparities in the their diagnoses of sinusitis and URI. The study methodology did not permit further quantification of this disparity. This spread could reflect important differences among these physicians in their belief systems about respiratory tract infections. It might also reflect differences in coding practices or other aspects of physician practice style. Further investigation will be necessary to understand the decision-making practices of this subset of physicians.

The retrospective methodology used in this study posed some challenges. The data depended on the information that the physicians chose to record in the progress notes. It was our assumption that the physicians were likely to have recorded those clinical factors they considered to be most pertinent to their decision making. Under these assumptions, it is quite interesting to compare the actual practice patterns of physicians with their responses to the previous survey methods that used simulated cases. We believe the results of the studies showed a reasonable level of consistency for the key clinical factors while revealing new information about the importance of a history of sinusitis in determining the diagnosis and management of cases seen in practice.

The results of this study could be limited by the fairly small convenience sample of local family physicians. Local training influences might be reflected in the management of these patients. As a result, the generalizability of these findings might be limited.

In summary, this study showed that patients are more likely to have sinusitis diagnosed if they have discolored rhinorrhea, facial pain, headache, or a history of sinusitis. Findings of sinus tenderness or purulent nasal discharge also contribute to this diagnosis. These findings are based on clinical practice, and they are generally consistent with responses to simulated cases and with a previous regression analysis. Further work in this area is necessary to understand the beliefs of physicians with large disparities in the diagnosis of sinusitis and to observe future trends in antibiotic use for patients with sinusitis and other respiratory tract infections.

\section{References}

1. van Duijn NP, Brouwer HJ, Lamberts H. Use of symptoms and signs to diagnose maxillary sinusitis in 
general practice: comparison with ultrasonography. BMJ 1992;305:684-7.

2. Williams JW Jr, Simel DL, Roberts L, Samsa GP. Clinical evaluation for sinusitis: Making the diagnosis by history and physical examination. Ann Intern Med 1992;117:705-10.

3. Hickner JM. Predicting acute maxillary sinusitis. J Fam Pract 1996;42:222-3.

4. Little DR, Mann BL, Sherk DW. Factors influencing the clinical diagnosis of sinusitis. J Fam Pract 1998;46:147-52.

5. Gwaltney JM Jr, Sydnor A. Jr, Sande MA. Etiology and antimicrobial treatment of acute sinusitis. Ann Otol Rhinol Laryngol Suppl 1981;90(3 Pt 3):68-71.

6. Evans FO Jr, Sydnor JB, Moore WE, et al. Sinusitis of the maxillary antrum. N Engl J Med 1975;293: 735-9.

7. Gordts F, Clement PA, Buisseret T. Prevalence of sinusitis signs in a non-ENT population. ORL J Otorhinolaryngol Relat Spec 1996;58:315-9.

8. Lindbaek $M$, Hjortdahl $P$, Johnsen UL. Use of symptoms, signs, and blood tests to diagnose acute sinus infections in primary care: comparison with computed tomography. Fam Med 1996;28:183-8.

9. Hansen JG, Schmidt H, Rosborg J, Lund E. Predict- ing acute maxillary sinusitis in a general practice population. BMJ 1995;311:233-6.

10. Diagnosis and treatment of acute bacterial rhinosinusitis summary, Evidence report/technology assessment, number 9. AHCPR pub no 99-E015. Rockville, Md: Agency for Health Care Policy and Research. March 1999. http://www.ahcpr.gov/clinic/ sinussum.htm.

11. Little DR. Predicting sinusitis. J Fam Pract 1996;43: 113-4.

12. Hueston WJ, Eberlein C, Johnson D, Mainous AG 3 rd. Criteria used by clinicians to differentiate sinusitis from viral upper respiratory tract infection. $J$ Fam Pract 1998;46:487-92.

13. Stalman W, van Essen GA, van der Graaf $Y$, de Melker RA. Maxillary sinusitis in adults: an evaluation of placebo-controlled double-blind trials. Fam Pract 1997;14:124-9.

14. Hickner JM. Antibiotics for acute maxillary sinusitis in adults. J Fam Pract 1998;46:281-2.

15. Hamm RM, Hicks RJ, Bemben DA. Antibiotics and respiratory infections: are patients more satisfied when expectations are met? J Fam Pract 1996;43:5662. 